\title{
INVISIBLE HIGGS DECAYS FROM HIGGS GRAVISCALAR MIXING
}

\author{
Daniele Dominici, ${ }^{1, *}$ and John F. Gunion ${ }^{2}$, 田 \\ ${ }^{1}$ Department of Physics, University of Florence, Sesto F. (FI), \\ Florence and Istituto Nazionale di Fisica Nucleare, Sezione di Firenze, Italy \\ ${ }^{2}$ Department of Physics, University of California at Davis, Davis, CA 95616
}

(Dated: December 2, 2018)

\begin{abstract}
We recompute the invisible Higgs decay width arising from Higgs-graviscalar mixing in the ADD model, comparing the original derivation in the non-diagonal mass basis to that in a diagonal mass basis. The results obtained are identical (and differ by a factor of 2 from the original calculation) but the diagonal-basis derivation is pedagogically useful for clarifying the physics of the invisible width from mixing. We emphasize that both derivations make it clear that a direct scan in energy for a process such as $W W \rightarrow W W$ mediated by Higgs plus graviscalar intermediate resonances would follow a single Breit-Wigner form with total width given by $\Gamma^{t o t}=\Gamma_{h}^{S M}+\Gamma_{\text {invisible }}$. We also compute the additional contributions to the invisible width due to direct Higgs to graviscalar pair decays. We find that the invisible width due to the latter is relatively small unless the Higgs mass is comparable to or larger than the effective extra-dimensional Planck mass.
\end{abstract}

\section{INTRODUCTION}

In several extensions of the Standard Model (SM) there exist mechanisms which modify the Higgs decay rates in channels observable at the LHC. One recent example is the Randall Sundrum model [1] where the Higgs-radion mixing can modify Higgs production and decay at the LHC [2, 3]. These effects may be detected both through a reduction in the Higgs yield and in the direct observation of radion decays [2, 3, 44]. There are also examples where the reduction comes from a substantial invisible width, as occurs for example in those supersymmetric models in which the Higgs has a large branching ratio into the lightest gravitinos or neutralinos. Invisible decay of the Higgs is also predicted in models with large extra dimensions felt by gravity (ADD) [5, 6], our focus in this paper.

In ADD models, the presence of an interaction between the Higgs $H$ and the Ricci scalar curvature of the induced 4-dimensional metric, $g_{\text {ind }}$, given by the action [7]

$$
S=-\xi \int d^{4} x \sqrt{g_{\text {ind }}} R\left(g_{\text {ind }}\right) H^{\dagger} H
$$

generates, after the usual shift $H=((v+h) / \sqrt{2}, 0)$, the mixing term

$$
\mathcal{L}_{m i x}=-\frac{2 \xi v M_{H}^{2}}{M_{P}} \sqrt{\frac{3(\delta-1)}{\delta+2}} h \Sigma_{\vec{n}} \phi_{G}^{\vec{n}}
$$

where the $\phi_{G}^{\vec{n}}$ are complex graviscalar fields, $M_{P}$ is the reduced Planck mass $\left(M_{P}=\left(8 \pi G_{N}\right)^{-1 / 2}\right), \delta$ is the number of extra dimensions and $\xi$ is a dimensionless parameter. Noting that hermiticity requires $\phi_{G}^{\vec{n}}=\left[\phi_{G}^{-\vec{n}}\right]^{*}$ and writing $\phi_{G}^{\vec{n}}=\frac{1}{\sqrt{2}}\left(s_{\vec{n}}+i a_{\vec{n}}\right)$, we may restrict the sums to $\vec{n}>0$, by which we mean the first non-zero entry of $\vec{n}$ is positive. ${ }^{1}$ Then, defining

$$
\epsilon \equiv-\frac{2 \sqrt{2}}{M_{P}} \xi v m_{h}^{2} \sqrt{\frac{3(\delta-1)}{\delta+2}}
$$

one obtains

$$
\mathcal{L}_{\text {mix }}=\epsilon h \sum_{\vec{n}>0} s_{\vec{n}}
$$

\footnotetext{
*dominici@fi.infn.it

$1 \quad$ It is quite crucial to explicitly keep only $\vec{n}>0$ states since $s_{\vec{n}<0}$ is not independent of $s_{\vec{n}>0}$. This is especially important for obtaining correct Feynman rules that avoid double counting.
} 
where $s_{\vec{n}}$ is a CP-even canonically normalized graviscalar KK excitation with mass $m_{\vec{n}}^{2}=4 \pi^{2} \vec{n}^{2} / L^{2}, L$ being the size of each of the extra dimensions.

As a result of the above mixing, instead of a single Higgs boson, one must consider the production of the full set of densely spaced mass eigenstates all of which are mixing with one another. The new signature that arises as a result of this mixing is that the Higgs boson will effectively acquire a possibly very large branching ratio to invisible final states composed primarily of graviscalars. The purpose of this paper is to first rederive the result obtained in [7, 8] comparing the non-diagonal mass and diagonal mass bases in the direct Feynman diagram approach. Our approach clarifies the nature of this effect and also reveals a factor two error in the original derivation (as confirmed in [9]). (A brief summary of our results and related phenomenology appeared in [10].) These computations set the stage for our main goal of computing the Higgs to graviscalar pair width, in particular making it clear that such computations are most easily performed using the non-diagonal (Lagrangian) basis states rather than the mass eigenstate basis. The graviscalar pair states add to the invisible width coming purely from Higgs-graviscalar mixing. As we show, this additional invisible width is small relative to the mixing width if $m_{h}$ is small compared to $M_{D}\left(M_{D}\right.$ is related to the $D$ dimensional reduced Planck constant $\bar{M}_{D}$ by $\left.M_{D}=(2 \pi)^{\delta /(2+\delta)} \bar{M}_{D}\right)$ but should be accounted for in any eventual precision comparison between theory and experiment if $m_{h}$ is comparable to or larger than $M_{D}$.

\section{INVISIBLE WIDTH}

In [7, 8] the invisible Higgs width is calculated by extracting the imaginary part of the Higgs self energy, including the effects of Higgs-graviscalar mixing. The result we obtain following this general approach differs by a factor of two from that of [7, [8] (basically because of the need to use properly normalized $s_{\vec{n}}$ states) and is given by

$$
\Gamma_{i n v}\left(h \rightarrow s_{\vec{n}}\right)=2 \pi \xi^{2} v^{2} \frac{3(\delta-1)}{\delta+2} \frac{m_{h}^{1+\delta}}{M_{D}^{2+\delta}} S_{\delta-1} \sim(16 M e V) 20^{2-\delta} \xi^{2} S_{\delta-1} \frac{3(\delta-1)}{\delta+2}\left(\frac{m_{h}}{150 G e V}\right)^{1+\delta}\left(\frac{3 T e V}{M_{D}}\right)^{2+\delta}
$$

where $S_{\delta-1}=2 \pi^{\delta / 2} / \Gamma(\delta / 2)$ denotes the surface of a unit radius sphere in $\delta$ dimensions. In this paper, we first repeat the derivation of this result in the $h-s_{\vec{n}}$ basis, i.e. before mass diagonalization. In our second derivation, we first diagonalize the Hamiltonian to obtain the mass eigenstates. In both cases, we compute, by way of example, the amplitude for $W W \rightarrow W W$ scattering coming from summing over the diagonal Higgs and graviscalar eigenstate exchanges. The derivations make it absolutely clear that a scan of the cross section for $W W \rightarrow W W$ scattering over $s_{W W}$ would reveal a simple Breit-Wigner of width $\Gamma_{h}^{S M}+\Gamma_{\text {invisible }}$, implying that a direct scan in $s_{W W}$ can be used to determine $\Gamma_{\text {inv }}$. Further, after integrating over energy, the invisible width suppresses the LHC Higgs rate in the standard observable channels (such as $W W$ ) by a factor of $1 /(1+R)$ where

$$
R \equiv \frac{\Gamma_{\text {invisible }}}{\Gamma_{h}^{S M}}
$$

can be quite substantial even for a Higgs boson with mass above the $W W$ decay threshold. These two different ways of determining $R$ can then be checked for consistency. In contrast, as pointed out in [7], a process such as $e^{+} e^{-} \rightarrow Z^{*} \rightarrow Z+X$ cannot be directly employed to determine $R$ by simply measuring the ratio of the $X=$ invisible rate relative to the $X=$ visible rate.

\section{A. Derivation of the invisible width from graviscalar insertions into the Higgs propagator}

It is useful to first present a derivation of the above expression for the invisible Higgs width following a procedure that is essentially that of Refs. [7, 8]. We begin with the expression for the mixing Lagrangian given in Eq. (4). We consider a process such as $W W \rightarrow W W$ and recall that only the $h$ states have significant (i.e. not suppressed by $\left.1 / M_{P}\right)$ coupling to $W W$. The contributing Feynman diagrams are such that one begins with a $W W \rightarrow h$ vertex and ends with an $h \rightarrow W W$ vertex. There are then diagrams with no $\sum_{\vec{n}>0} s_{\vec{n}}$ insertions, one $\sum_{\vec{n}>0} s_{\vec{n}}$ insertion with two mixing vertices and so forth, resulting in a geometric series that can be resummed to give an effective $W W \rightarrow W W$ s-channel scattering amplitude with exactly the same form including normalization as a single Higgs exchange but with an additional contribution to the self-energy of the Higgs such that

$$
\mathcal{A}_{W W \rightarrow W W}=\frac{g_{W W h}^{2}}{s_{W W}-m_{h}^{2}+i m_{h} \Gamma_{h}^{S M}} \rightarrow \frac{g_{W W h}^{2}}{s_{W W}-m_{h}^{2}+i m_{h} \Gamma_{h}^{S M}+\Sigma\left(s_{W W}\right)}
$$


where

$$
\operatorname{Im} \Sigma(s)=-\epsilon^{2} \operatorname{Im} \sum_{\vec{n}>0} \frac{1}{s-m_{n}^{2}+i \epsilon} \rightarrow-\epsilon^{2} \frac{1}{4} \frac{M_{P}^{2}}{M_{D}^{2+\delta}} S_{\delta-1}(-\pi) s^{(\delta-2) / 2}=2 \pi \frac{3(\delta-1)}{\delta+2} \xi^{2} v^{2} m_{h}^{2} \frac{s^{(\delta-2) / 2}}{M_{D}^{2+\delta}} S_{\delta-1} .
$$

This is interpreted as saying that the Higgs has acquired an additional width given by

$$
\Gamma_{\text {invisible }}=\frac{1}{m_{h}} \operatorname{Im} \Sigma\left(m_{h}^{2}\right)=2 \pi \frac{3(\delta-1)}{\delta+2} \xi^{2} v^{2} \frac{m_{h}^{1+\delta}}{M_{D}^{2+\delta}} S_{\delta-1}
$$

that is deemed an invisible width since the $s_{\vec{n}}$ graviscalar states do not interact with ordinary matter and would be invisible in a detector.

The procedure for deriving the expression following the arrow in Eq. (8) is as follows. First, one converts the sum over $\vec{n}>0$ to an integral over a continuous spectrum of $s_{\vec{n}}$ masses as follows:

$$
\sum_{\vec{n}>0} \frac{1}{s-m_{n}^{2}+i \epsilon} \rightarrow \frac{1}{2} \int d m^{2} \rho_{\delta}(m) \frac{1}{s-m^{2}+i \epsilon}=\frac{1}{4} \frac{M_{P}^{2}}{M_{D}^{2+\delta}} S_{\delta-1} \int d m^{2} \frac{m^{\delta-2}}{s-m^{2}+i \epsilon}
$$

where the factor of $1 / 2$ arises in going from $\sum_{\vec{n}>0}$ to $\sum_{\vec{n}}$ and we have used the following expression for the state density:

$$
\rho_{\delta}(m)=\frac{L^{\delta} m^{\delta-2}}{(4 \pi)^{\delta / 2} \Gamma(\delta / 2)}=\frac{M_{P}^{2}}{M_{D}^{2+\delta}} \frac{\pi^{\delta / 2}}{\Gamma(\delta / 2)} m^{\delta-2}=\frac{1}{2} \frac{M_{P}^{2}}{M_{D}^{2+\delta}} S_{\delta-1} m^{\delta-2}
$$

with $L^{\delta}=(2 \pi)^{\delta} \frac{M_{P}^{2}}{M_{D}^{2+\delta}}$. Then, using

$$
\operatorname{Im} \frac{1}{s-m^{2}+i \epsilon}=-\pi \delta\left(s-m^{2}\right)
$$

one has

$$
\operatorname{Im} \sum_{\vec{n}>0} \frac{1}{s-m_{n}^{2}+i \epsilon} \rightarrow \frac{1}{4} \frac{M_{P}^{2}}{M_{D}^{2+\delta}} S_{\delta-1}(-\pi) s^{(\delta-2) / 2} .
$$

We emphasize that Eq. (77) implies that a scan of the cross section for $W W \rightarrow W W$ as $s_{W W}$ is varied would reveal a single Breit-Wigner of width $\Gamma_{h}^{S M}+\Gamma_{\text {invisible }}$ to the extent that the real part of $\Sigma\left(s_{W W}\right)$ can be neglected. However, the real part of $\Sigma(s)$ can lead to mass and wave function renormalization (see Appendix), which corrections are of order $m_{h}^{4} / M_{D}^{4}$. As a result, the apparent magnitude of the total width measured in a scan will receive corrections of this order. In addition, the interpretation of the normalization of $\mathcal{A}_{W W \rightarrow W W}$ (or any other process beginning with SM particles and ending with SM particles) and of the effective pole location will be similarly affected. In addition, our focus here, we find that there are further corrections to the effective width coming from additional contributions to $\operatorname{Im} \Sigma$. In particular, we will discuss the $h \rightarrow s_{\vec{n}} s_{\vec{m}}$ type of insertions. These we will find to be of order $\xi^{2}\left(m_{h} / M_{D}\right)^{2+\delta}$ relative to the mixing width, and therefore potentially significant if $m_{h}>M_{D}$. However, in practice the invisible width from the $s_{\vec{n}} s_{\vec{m}}$ pair final states is suppressed sufficiently by two-body phase space that the pair-width to mixing-width ratio is typically very small.

Of course, the ratio $R$ of Eq. (6) can be large even when $\left(m_{h} / M_{D}\right)^{4}$ is small. To illustrate the possibilities for $R$ for typical parameter choices of interest, we give in Figs. 1 and 2 a few contour plots of $R$ as a function of $\xi$ and $m_{h}$ for fixed choices of $M_{D}$ and $\delta$. These plots make it clear that even if $m_{h}$ is small compared to $M_{D}$ a substantial invisible width relative to the SM width is possible for relatively modest values of $\xi$. In particular, a large value for $R$ is possible for quite small $\xi$ values $\left(\right.$ i.e. $<0.1$ ) when $m_{h}$ is below the $W W$ decay threshold.

\section{B. Diagonal basis derivation of the invisible width}

In this section, we summarize a direct Feynman rule based derivation of the invisible width, based on first diagonalizing the Hamiltonian. One begins with a Hamiltonian that includes the widths of the various states and the mixing between the states. Because of the widths, the Hamiltonian is intrinsically complex and nonHermitian. One must then diagonalize the Hamiltonian to obtain the mass eigenstates. This is the procedure 

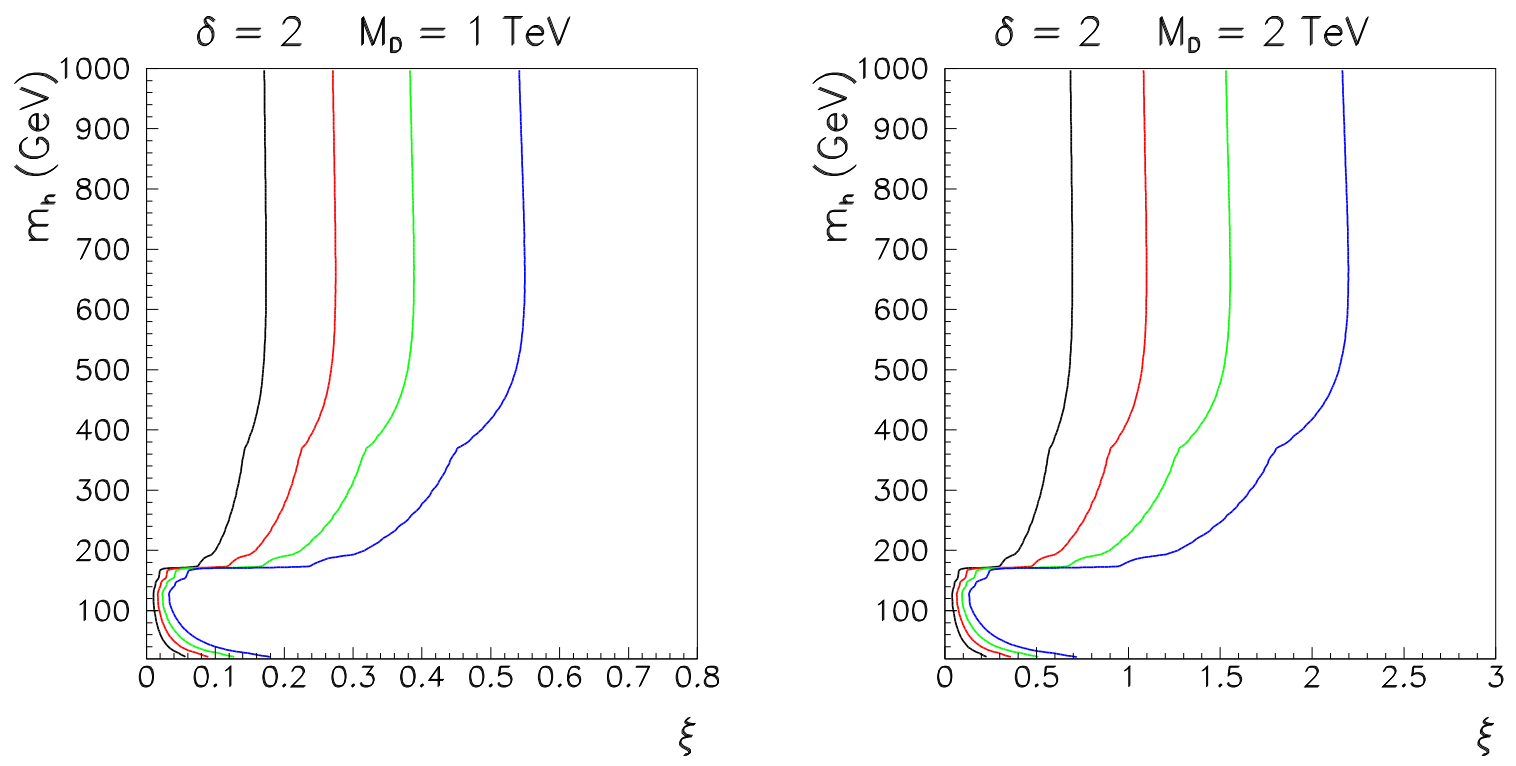

FIG. 1: We display contours of $R=0.1,0.25,0.5$ and 1 (from left to right) in the $m_{h}-\xi$ plane, for (left plot) $\delta=2$ and $M_{D}=1 \mathrm{TeV}$ and (right plot) $\delta=2$ and $M_{D}=2 \mathrm{TeV}$.
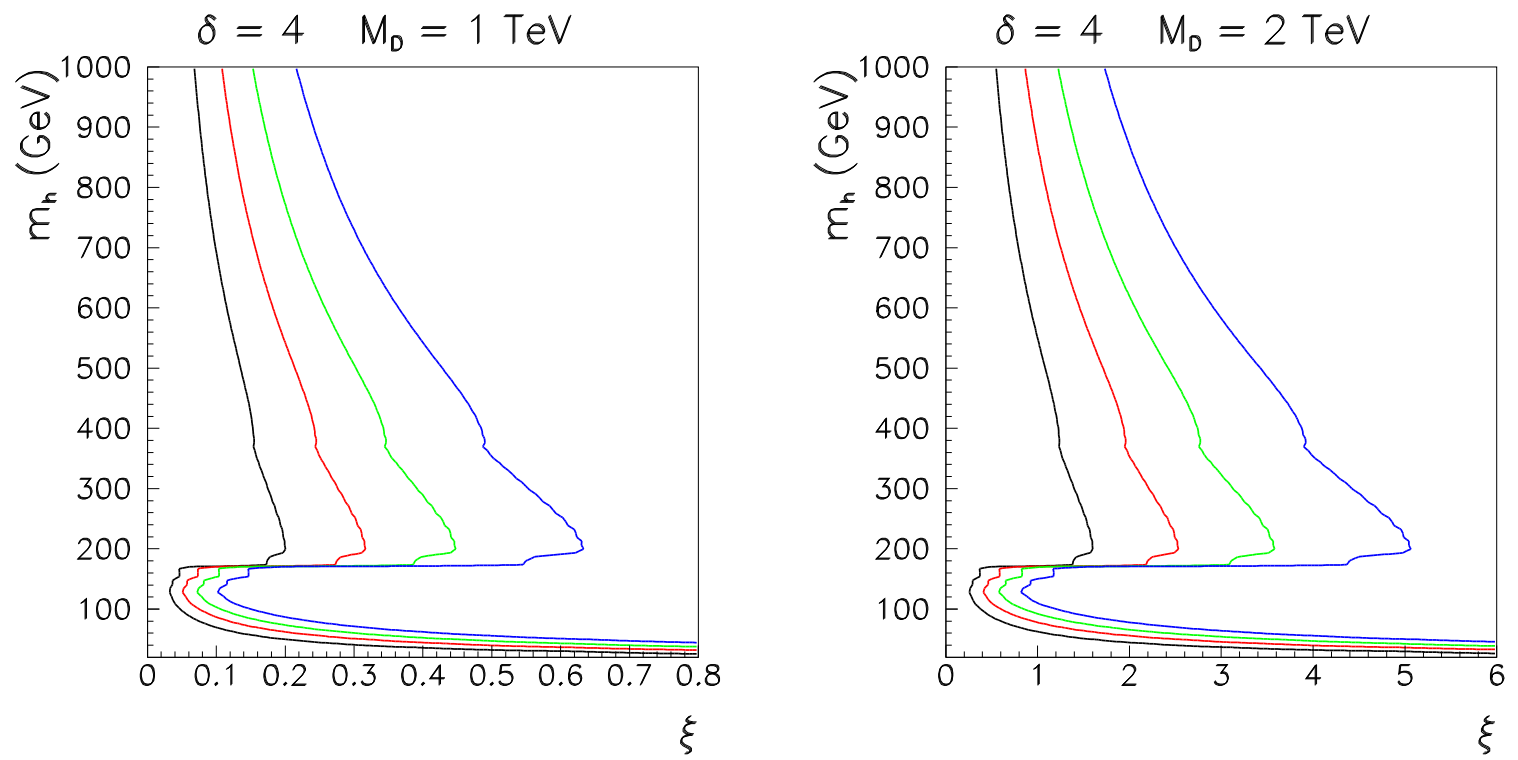

FIG. 2: We display contours of $R=0.1,0.25,0.5$ and 1 (from left to right) in the $m_{h}-\xi$ plane, for (left plot) $\delta=4$ and $M_{D}=1 \mathrm{TeV}$ and (right plot) $\delta=4$ and $M_{D}=2 \mathrm{TeV}$.

that is employed when studying the $K^{0}-\bar{K}^{0}$ system. From the diagonalized Hamiltonian one can then derive the Feynman rules and compute the $W W \rightarrow W W$ amplitude using them.

Since the $h$ is the only one of the states with couplings to $W W$ and $f \bar{f}$ (that are not suppressed by $1 / M_{P}$ ) it is the only state with a substantial inverse propagator imaginary component, $i m_{h} \Gamma_{h}^{S M}$. A crucial question is the size of $m_{h} \Gamma_{h}^{S M}$ relative to $\epsilon$ and relative to the spacing between the graviscalar states. For $m_{h} \sim 100 \mathrm{GeV}$ and $\Gamma_{h}^{S M} \sim$ few $\mathrm{MeV}, m_{h} \Gamma_{h}^{S M} \sim 0.1 \mathrm{GeV}^{2}$. The spacing between graviscalar states depends upon $\delta$, ranging from $\sim \mathrm{eV}$ for $\delta=2$ to $0.1 \mathrm{GeV}$ for $\delta=8$. The smallest spacing in mass-squared (taking $\delta=2$ ) is of order $100 \mathrm{GeV} \times 1 \mathrm{eV} \sim 10^{-7} \mathrm{GeV}^{2}$, so that it will be important to understand what happens when the $h$ and $s_{i}$ states are essentially degenerate. The magnitude of $\epsilon$ is $\sim(100 \mathrm{GeV})^{3} / 10^{19} \mathrm{GeV} \sim 10^{-13} \mathrm{GeV}^{2}$, i.e. much smaller than 
either the smallest mass-squared splitting or $m_{h} \Gamma_{h}^{S M}$. This fact will enter implicitly into some of our expansions.

The relevant mass-squared matrix is (using the Lagrangian form and defining $\rho \equiv m_{h} \Gamma_{h}^{S M}$ )

$$
\mathcal{L} \propto-\frac{1}{2}\left(m_{h}^{2}-i \rho\right) h^{2}-\frac{1}{2} \sum_{\vec{n}>0} m_{\vec{n}}^{2} s_{\vec{n}}^{2}+\epsilon h \sum_{\vec{n}>0} s_{\vec{n}}
$$

and can be diagonalized to order $\epsilon^{2}$ by the following transformations:

$$
\begin{gathered}
h=N\left[h^{\prime}+\sum_{\vec{m}>0} \frac{\epsilon}{m_{h}^{2}-i \rho-m_{\vec{m}}^{2}} s_{\vec{m}}^{\prime}\right] \\
s_{n}=N_{\vec{n}}\left[s_{\vec{n}}^{\prime}-\frac{\epsilon}{m_{h}^{2}-i \rho-m_{\vec{n}}^{2}} h^{\prime}-\frac{1}{2} \frac{\epsilon^{2}}{\left(m_{\vec{n}}^{2}-m_{h}^{2}+i \rho\right)} \sum_{\vec{m} \neq \vec{n}, \vec{n}>0, \vec{m}>0} \frac{1}{m_{\vec{m}}^{2}-m_{h}^{2}+i \rho} s_{\vec{m}}^{\prime}\right]
\end{gathered}
$$

where $h$ and $s_{n}$ are the original fields before diagonalizing the Hamiltonian and

$$
N=\left[1+\sum_{\vec{m}>0} \frac{\epsilon^{2}}{\left(m_{h}^{2}-i \rho-m_{\vec{m}}^{2}\right)^{2}}\right]^{-1 / 2}, \quad N_{\vec{n}}=\left[1+\frac{\epsilon^{2}}{\left(m_{h}^{2}-i \rho-m_{\vec{n}}^{2}\right)^{2}}\right]^{-1 / 2} .
$$

The corresponding mass squared eigenvalues are

$$
m_{h^{\prime}}^{2}=m_{h}^{2}-i \rho+\epsilon^{2} \sum_{\vec{n}>0} \frac{1}{m_{h}^{2}-i \rho-m_{\vec{n}}^{2}}, \quad m_{s_{\vec{n}}^{\prime}}^{2}=m_{\vec{n}}^{2}-\epsilon^{2} \frac{1}{m_{h}^{2}-i \rho-m_{\vec{n}}^{2}} .
$$

The $\mathcal{A}(W W \rightarrow W W)$ amplitude is then obtained as the sum $\mathcal{A}\left(W W \rightarrow h^{\prime} \rightarrow W W\right)+\sum_{\vec{n}>0} \mathcal{A}\left(W W \rightarrow s_{\vec{n}}^{\prime} \rightarrow\right.$ $W W$ ) and takes the following form, as shown in Appendix $\mathrm{A}$.

$$
\begin{aligned}
& \mathcal{A}_{W W \rightarrow h^{\prime} \rightarrow W W}+\sum_{\vec{n}>0} \mathcal{A}_{W W \rightarrow s_{\vec{n}}^{\prime} \rightarrow W W} \\
\sim & \frac{i}{p^{2}-m_{h}^{2}+i \rho+\sum_{\vec{n}>0} \frac{\epsilon^{2}}{m_{\vec{n}}^{2}-m_{h}^{2}+i \rho}}\left(1-\frac{1}{2} \sum_{\vec{n}>0} \frac{\epsilon^{2}}{\left(m_{\vec{n}}^{2}-m_{h}^{2}+i \rho\right)^{2}}\right)^{2}+\sum_{\vec{n}>0} \frac{i}{p^{2}-m_{\vec{n}}^{2}-\frac{\epsilon^{2}}{m_{\vec{n}}^{2}-m_{h}^{2}+i \rho}}\left(\frac{-\epsilon}{m_{\vec{n}}^{2}-m_{h}^{2}+i \rho}\right)^{2} \\
\sim & \frac{i}{p^{2}-m_{h}^{2}+i \rho-\sum_{\vec{n}>0} \frac{\epsilon^{2}}{p^{2}-m_{\vec{n}}^{2}}} \simeq \frac{i}{s-m_{h}^{2}+i m_{h}\left(\Gamma_{h}^{S M}+\Gamma_{\mathrm{inv}}\right)} .
\end{aligned}
$$

From the final expression we see (again) that the behavior of the $W W \rightarrow W W$ scattering amplitude is indeed that obtained by replacing the SM Higgs width, $\Gamma_{h}^{S M}$, by $\Gamma_{h}^{S M}+\Gamma_{\text {inv }}$ in the self energy portion of the Higgs propagator, with $\Gamma_{\text {inv }}$ as given in Eq. (8). We also stress that this form implies that the effective cross section for $W W \rightarrow W W$ from $s$-channel Higgs resonance exchange will be suppressed compared to that predicted in the SM by a factor of $1 /(1+R)$.

Of course, it should be stressed that all of these same remarks apply to any process where the Higgs is exchanged in the $s$-channel beginning with a SM state and ending with a SM state. In any such channel in which one can scan over the Higgs resonance, the width of the resonance will be $\Gamma_{h}^{S M}+\Gamma_{\text {inv }}$ instead of $\Gamma_{h}^{S M}$ and the net cross section will be suppressed compared to the SM prediction by $1 /(1+R)$. As studied in [11], there are many indirect and direct techniques for measuring the total width of the Higgs resonance. These range from the very precise determinations by direct scanning in $\sqrt{s}$ at a muon collider, which yields excellent accuracy for the width even for a very narrow Higgs as found for $m_{h} \sim 100-150 \mathrm{GeV}$, to looking at $g g \rightarrow h \rightarrow Z Z^{(*)} \rightarrow 4 \ell$ at a hadron collider, where a direct scan determination of the Higgs width is possible for $m_{h} \gtrsim 200 \mathrm{GeV}$, i.e. whenever the width is larger than a couple of $\mathrm{GeV}$. Of course, if $\Gamma_{h}^{S M}+\Gamma_{\text {inv }} \gg \Gamma_{h}^{S M}$, then the latter will be possible down to considerably lower values of $m_{h}$. For example, from Fig. 团 we see that $\xi \sim 0.6$ will give $\Gamma_{h}^{S M}+\Gamma_{\mathrm{inv}} \sim 2 \mathrm{GeV}$ at $m_{h}=120 \mathrm{GeV}$ for $\delta=2$ and $M_{D}=1 \mathrm{TeV}$. At a next-generation linear collider, the best technique for directly scanning the Higgs resonance is to look at the Higgs peak shape in $e^{+} e^{-} \rightarrow Z X$ as a function of $M_{X}$. This again works in the case of a SM-like width $\Gamma_{h}^{S M}$ down to $m_{h} \sim 200 \mathrm{GeV}$, extending to substantially lower values for any case where $\Gamma_{h}^{S M}+\Gamma_{\mathrm{inv}} \gtrsim 2 \mathrm{GeV}$. In the absence of a muon collider, Ref. [11] details the means for measuring the effective Higgs width for cases where $\Gamma_{h}^{S M}+\Gamma_{\text {inv }} \lesssim 1 \mathrm{GeV}$ using a subtle combination of $\gamma \gamma$ collider data and $e^{+} e^{-}$collider data. 


\section{Additional contributions to the invisible state production coming from direct two-graviscalar production processes}

In the above, it was assumed that the only way in which invisible intermediate states contribute to $W W \rightarrow W W$ near the Higgs resonance is through multiple iterations of $h \rightarrow s_{\vec{n}} \rightarrow h$ type mixing. However, there are other contributing invisible intermediate states as a result of the presence of $W W \rightarrow s_{\vec{k}} s_{\vec{l}}$ processes. First, there are $W W s_{\vec{k}} s_{\vec{l}}$ contact interactions. Second, there are the $s$-channel exchange processes involving $W W \rightarrow h+s_{\vec{n}} \rightarrow s_{\vec{k}} s_{\vec{l}}$. (Here, non-s-channel diagrams could also be included, but would yield very small contributions compared to those we consider, assuming reasonable resolution in the final state.) To include the additional resonant sources of invisible state production requires a significant calculation, performed below. We also show that the $s_{\vec{n}} s_{\vec{k}} s_{\vec{l}}$ vertices that are present in the theory do not contribute to the process at hand. Note that we have found it easiest to perform this calculation in the $h, s_{\vec{n}}$ basis rather than in the diagonalized $h^{\prime}, s_{\vec{n}}^{\prime}$ basis. In part, this is because it is the $s_{\vec{n}}$ 's that are truly invisible. But also, the $h-s_{\vec{n}}$ basis is simply easier to use, just as was the case for the mixing width calculation. At the end, we find that, to a good approximation, the appropriate comparison is the contribution to the Higgs invisible width coming from $h \rightarrow s_{\vec{n}} s_{\vec{m}}$ decays to that coming from $h-s_{\vec{n}}$ mixing.

Let us first discuss the $W W s_{\vec{k}} s_{\vec{l}}$ contact interactions. These derive from expanding the interaction Lagrangian to order $\kappa^{2}$ where $\kappa=2 / M_{D}^{1+\delta / 2}$. This expansion takes the form

$$
\begin{aligned}
\int d^{4} x \int d y \sqrt{-\widehat{g}} \mathcal{L}(\widehat{g})=\int & d^{4} x \int d y \delta(y)\left\{\left[\left.\mathcal{L}(\widehat{g})\right|_{\widehat{g}=\eta}-\frac{\kappa}{2} h^{\mu \nu} T_{\mu \nu}\right]\right. \\
& +\kappa^{2}\left[\left.A \mathcal{L}(\widehat{g})\right|_{\widehat{g}=\eta}-\left.B^{\mu \nu} \frac{\delta \mathcal{L}}{\delta \widehat{g}^{\mu \nu}}\right|_{\widehat{g}=\eta}\right] \\
& \left.+\kappa^{2} h^{\mu \nu}(x, y)\left[\left.\frac{1}{2} \int d^{4} x^{\prime} \frac{\delta^{2} \mathcal{L}}{\delta \widehat{g}^{\mu \nu} \delta \widehat{g}^{\rho \sigma}}\right|_{\widehat{g}=\eta} h^{\rho \sigma}\left(x^{\prime}, y\right)\right]\right\}
\end{aligned}
$$

where

$$
A=\frac{1}{8} h^{2}-\frac{1}{4} h_{\rho \sigma} h^{\rho \sigma}, \quad B^{\mu \nu}=\frac{1}{2} h h^{\mu \nu}-h^{\mu \lambda} h_{\lambda}^{\nu}, \quad h=h_{\mu}^{\mu} .
$$

After employing the $\delta(y)$, which gives a factor of $1 / V^{\delta}$, using the identification $\frac{\kappa^{2}}{V^{\delta}}=1 / M_{P}^{2}$, and using the fact that for an initial $W W$ state we would have $\left.\frac{\delta \mathcal{L}}{\delta \widehat{g}^{\mu \nu}}\right|_{\widehat{g}=\eta} \sim m_{W}^{2} W^{\mu} W_{\mu}+\ldots$, we find an amplitude contribution to $W W \rightarrow s_{\vec{k}} s_{\vec{l}}$ that is $\propto m_{W}^{2} / M_{P}^{2}$. Squaring and integrating over a window of $d s$ of size $\sim m_{h} \Gamma_{\text {res }}$, we get a cross section contribution of order

$$
\frac{m_{W}^{4}}{M_{D}^{8}} m_{h}^{5} \Gamma_{\text {res }}
$$

This can be compared to the $s$-channel $h$ exchange contribution which gives an integrated cross section for $W W \rightarrow h \rightarrow W W$ of rough size (assuming that the resolution window size $\Gamma_{\text {res }}$ is substantially larger than $\Gamma_{h}^{S M}$ )

$$
g^{4} m_{W}^{4} \frac{\pi}{m_{h} \Gamma_{h}^{S M}} .
$$

The ratio of the contact to the $s$-channel contribution is then very roughly given by

$$
\frac{m_{h}^{6} \Gamma_{h}^{S M} \Gamma_{\text {res }}}{\pi g^{4} M_{D}^{8}}
$$

This ratio will typically be very small provided $\Gamma_{\text {res }}$ is of order a few $\mathrm{GeV}$ and $M_{D}>1 \mathrm{TeV}$.

Now let us turn to the cubic interactions that can lead to $W W \rightarrow h+s_{\vec{n}} \rightarrow s_{\vec{k}} s_{\vec{l}}$ type processes. To do so, we must go to the full $\mathcal{L}$ including all effects of the mixing term at the cubic level. A first source of such cubic interactions comes from the expansion of the Hilbert-Einstein Lagrangian up to the cubic order in the graviscalar fields. There are a huge number of terms, but after integrating over the extra dimensions one finds that all cubic $s_{\vec{n}} s_{\vec{k}} s_{\vec{l}}$ vertices are proportional to $\delta_{\vec{n}+\vec{k}+\vec{l}}$. This $\delta$ function implies that $|\vec{k}|+|\vec{l}| \geq|\vec{n}|$. Since $m_{\vec{m}} \propto|\vec{m}|$, this implies that $m_{\vec{k}}+m_{\vec{l}} \geq m_{\vec{n}}$. However, for the decay $s_{\vec{n}} \rightarrow s_{\vec{k}}+s_{\vec{l}}$ to be possible requires $m_{\vec{k}}+m_{\vec{l}}<m_{\vec{n}}$. Thus, there is no phase space available for the decays that could be mediated by these cubic vertices. 
The $h s_{\vec{n}} s_{\vec{k}}$ vertices that can mediate the decays of interest derive from the extra $T_{\mu}^{\mu}$ contributions that emerge from $\mathcal{L}_{\text {mix }}$ which take the form (using $H=\frac{1}{\sqrt{2}}(v+h)$ )

$$
T_{\mu}^{\mu e x t r a}=6 \xi \square\left(H^{\dagger} H\right),
$$

where

$$
\square\left(H^{\dagger} H\right)=\left(v \square h+h \square h+\partial_{\mu} h \partial^{\mu} h\right) .
$$

Including this full structure in the expression for $\mathcal{L}_{\text {int }}$, gives rise to an interaction term of the form:

$$
-\frac{\epsilon}{6 \xi v m_{h}^{2}} \sum_{\vec{n}>0} s_{\vec{n}}\left[4 V(h)-\partial^{\mu} h \partial_{\mu} h+6 \xi\left(v \square h+h \square h+\partial_{\mu} h \partial^{\mu} h\right)\right],
$$

where the full form for $V(h)$ is

$$
V(h)=\frac{1}{2} m_{h}^{2} h^{2}+\frac{1}{2} \frac{1}{v} m_{h}^{2} h^{3}+\frac{1}{8} \frac{1}{v^{2}} h^{4} .
$$

Thus, the full $\mathcal{L}$ takes the form:

$$
\mathcal{L}=-\frac{1}{2} \sum_{\vec{n}>0} s_{\vec{n}}\left(\square+m_{\vec{n}}^{2}\right) s_{\vec{n}}-\frac{1}{2} h \square h-\left(1+\sum_{\vec{n}>0} \frac{2}{3} \frac{\epsilon}{\xi v m_{h}^{2}} s_{\vec{n}}\right) V(h)-\frac{\epsilon}{v m_{h}^{2}}\left[v \square h+\partial_{\mu} h \partial^{\mu} h\left(1-\frac{1}{6 \xi}\right)+h \square h\right] \sum_{\vec{n}>0} s_{\vec{n}}
$$

or after partial integration

$$
\begin{aligned}
\mathcal{L}= & \frac{1}{2} \sum_{\vec{n}>0} \partial_{\mu} s_{\vec{n}} \partial^{\mu} s_{\vec{n}}-\frac{1}{2} \sum_{\vec{n}>0} m_{\vec{n}}^{2} s_{\vec{n}}^{2}+\frac{1}{2} \partial_{\mu} h \partial^{\mu} h-\left(1+\frac{2}{3} \frac{\epsilon}{\xi v m_{h}^{2}} \sum_{\vec{n}>0} s_{\vec{n}}\right) V(h) \\
& -\frac{\epsilon}{v m_{h}^{2}} \sum_{\vec{n}>0}\left(-v \partial_{\mu} h \partial^{\mu} s_{\vec{n}}-\partial^{\mu} s_{\vec{n}} h \partial_{\mu} h-\frac{1}{6 \xi} s_{\vec{n}} \partial^{\mu} h \partial_{\mu} h\right) .
\end{aligned}
$$

At this point, we must now employ the equations of motion for the $h$ and $s_{\vec{n}}$ fields. For the $s_{\vec{n}}$ we have

$$
\left(\square+m_{\vec{n}}^{2}\right) s_{\vec{n}}+\frac{\epsilon}{v m_{h}^{2}}\left(v \square h+\partial_{\mu} h \partial^{\mu} h+h \square h\right)+\frac{2}{3} \frac{\epsilon}{\xi v m_{h}^{2}}\left(V(h)-\frac{1}{4} \partial^{\mu} h \partial_{\mu} h\right)=0 .
$$

As regards the $h$, we have:

$$
\square h+\frac{\epsilon}{v m_{h}^{2}}(v+h) \sum_{\vec{n}>0} \square s_{\vec{n}}+\left(1+\frac{2 \epsilon}{3 \xi v m_{h}^{2}} \sum_{\vec{n}>0} s_{\vec{n}}\right) \frac{\partial V}{\partial h}+\frac{\epsilon}{3 \xi v m_{h}^{2}}\left[\partial^{\mu}\left(\sum_{\vec{n}>0} s_{\vec{n}} \partial_{\mu} h\right)\right]=0 .
$$

Returning to the original form of $\mathcal{L}$, given in Eq. (30), keeping only terms of cubic or quadratic order in fields, we get

$$
\begin{gathered}
\mathcal{L}=\frac{1}{2} \sum_{\vec{n}>0} \partial_{\mu} s_{\vec{n}} \partial^{\mu} s_{\vec{n}}-\frac{1}{2} \sum_{\vec{n}>0} m_{\vec{n}}^{2} s_{\vec{n}}^{2}+\frac{1}{2} \partial_{\mu} h \partial^{\mu} h-\frac{1}{2} m_{h}^{2} h^{2}-\frac{1}{2 v} m_{h}^{2} h^{3}-\frac{1}{3} \frac{\epsilon}{\xi v} \sum_{\vec{n}>0} s_{\vec{n}} h^{2} \\
-\frac{\epsilon}{v m_{h}^{2}}(v+h) \square h \sum_{\vec{n}>0} s_{\vec{n}}-\frac{\epsilon}{v m_{h}^{2}}\left(1-\frac{1}{6 \xi}\right) \partial^{\mu} h \partial_{\mu} h \sum_{\vec{n}>0} s_{\vec{n}} .
\end{gathered}
$$

We now make use of the equations of motion by substituting the expression for $\square h$ obtained using Eq. (32). First, we collect the purely quadratic terms in $\mathcal{L}$ from this substitution. This means we look for terms linear in the fields in $\square h$. To the needed order in $\epsilon$, we have

$$
(\square h)_{\text {linear }}=-m_{h}^{2} h-\sum_{\vec{m}>0} \frac{\epsilon}{m_{h}^{2}} \square s_{\vec{m}} .
$$

The relevant (quadratic) terms arise from the next to last term in $\mathcal{L}$ in Eq. (33) and are

$$
\mathcal{L}_{\text {quad }}^{\square}=\sum_{\vec{n}>0} \epsilon h s_{\vec{n}}+\sum_{\vec{n}>0} \sum_{\vec{m}>0} \frac{\epsilon^{2}}{m_{h}^{4}} s_{\vec{n}} \square s_{\vec{m}},
$$


the first being our standard mixing term. Important trilinear terms emerge from the second term of the next to last term in Eq. (33). Again keeping only $\mathcal{O}\left(\epsilon^{2}\right)$ or lower we have:

$$
\mathcal{L}_{\text {cubic }}^{\square}=-\frac{\epsilon}{v m_{h}^{2}} h \sum_{\vec{n}>0} s_{\vec{n}}(\square h)_{\text {linear }}-\sum_{\vec{n}>0} \frac{\epsilon}{m_{h}^{2}} s_{\vec{n}}(\square h)_{\text {quad }} .
$$

To the order needed,

$$
(\square h)_{\text {quad }}=-\frac{3}{2} \frac{m_{h}^{2}}{v} h^{2}-\sum_{\vec{m}>0} \frac{2 \epsilon}{3 \xi v} s_{\vec{m}} h-\sum_{\vec{m}>0} \frac{\epsilon}{v m_{h}^{2}} \square s_{\vec{m}} h-\frac{\epsilon}{3 v m_{h}^{2} \xi} \sum_{\vec{n}>0}\left(\partial^{\mu} s_{\vec{n}} \partial_{\mu} h+s_{\vec{n}} \square h\right)
$$

yielding

$$
\mathcal{L}_{\text {cubic }}^{\square}=\sum_{\vec{n}>0}\left[\frac{5}{2} \frac{\epsilon}{v} s_{\vec{n}} h^{2}+\frac{1}{3} \frac{\epsilon^{2}}{\xi v m_{h}^{2}} h s_{\vec{n}} \sum_{\vec{m}>0} s_{\vec{m}}+2 \frac{\epsilon^{2}}{v m_{h}^{4}} s_{\vec{n}} h \sum_{\vec{m}>0} \square s_{\vec{m}}+\frac{\epsilon^{2}}{3 v m_{h}^{4} \xi} s_{\vec{n}} \sum_{\vec{m}>0} \partial^{\mu} s_{\vec{m}} \partial_{\mu} h\right] .
$$

So, putting it all together, we find the following trilinear Lagrangian

$$
\begin{aligned}
\mathcal{L}_{\text {cubic }}=- & \frac{1}{2 v} m_{h}^{2} h^{3}-\frac{\epsilon}{3 \xi v} h^{2} \sum_{\vec{n}>0} s_{\vec{n}}-\frac{\epsilon}{v m_{h}^{2}}\left(1-\frac{1}{6 \xi}\right) \partial^{\mu} h \partial_{\mu} h \sum_{\vec{n}>0} s_{\vec{n}}+\mathcal{L}_{\text {cubic }}^{\square} \\
=- & \frac{1}{2 v} m_{h}^{2} h^{3}-\frac{\epsilon}{v m_{h}^{2}}\left(1-\frac{1}{6 \xi}\right) \partial^{\mu} h \partial_{\mu} h \sum_{\vec{n}>0} s_{\vec{n}}+\frac{\epsilon}{2 v}\left(5-\frac{2}{3 \xi}\right) h^{2} \sum_{\vec{n}>0} s_{\vec{n}} \\
& +\frac{1}{3} \frac{\epsilon^{2}}{\xi v m_{h}^{2}} h \sum_{\vec{n}>0} s_{\vec{n}} \sum_{\vec{m}>0} s_{\vec{m}}+2 \frac{\epsilon^{2}}{v m_{h}^{4}} h \sum_{\vec{n}>0} s_{\vec{n}} \sum_{\vec{m}>0} \square s_{\vec{m}}+\frac{\epsilon^{2}}{3 v m_{h}^{4} \xi} \sum_{\vec{n}>0} s_{\vec{n}} \sum_{\vec{m}>0} \partial^{\mu} s_{\vec{m}} \partial_{\mu} h
\end{aligned}
$$

of which it is the latter two terms that give the vertices of interest. The effective cubic Lagrangian for our purposes then becomes (after using $\square s_{\vec{m}} \rightarrow-m_{\vec{m}}^{2} s_{\vec{m}}$ and relabeling indices)

$$
\mathcal{L}_{\text {cubic }}=\epsilon^{2} \sum_{\vec{k}>0} \sum_{\vec{l}>0}\left[\frac{1}{2 \xi v m_{h}^{2}} h s_{\vec{l}} s_{\vec{k}}-\frac{2}{v m_{h}^{4}}\left(1-\frac{1}{6 \xi}\right) h s_{\vec{l}} m_{\vec{k}}^{2} s_{\vec{k}}\right] .
$$

The $h s_{\vec{k}} s_{\vec{l}}$ vertex (accounting for the many different Wick's contractions) takes the form $\epsilon^{2} g_{\vec{k} \vec{l}}$ where

$$
g_{\vec{k} \vec{l}}=\frac{1}{\xi v m_{h}^{2}}-\frac{2}{v m_{h}^{4}}\left(1-\frac{1}{6 \xi}\right)\left(m_{\vec{k}}^{2}+m_{\vec{l}}^{2}\right) .
$$

The invisible $h$ width arising from these interactions takes the form

$$
\Gamma(h \rightarrow \text { graviscalar pairs })=\frac{1}{2} \sum_{\vec{k}>0, \vec{l}>0} \frac{1}{16 \pi m_{h}^{3}}\left|\epsilon^{2} g_{\vec{l}, \vec{k}}\right|^{2} \lambda\left(m_{h}^{2}, m_{\vec{k}}^{2}, m_{\vec{l}}^{2}\right),
$$

where $\lambda(a, b, c)=\left[a^{2}+b^{2}+c^{2}-2 a b-2 a c-2 b c\right]^{1 / 2}$ is the usual two-body phase space factor and the $\frac{1}{2}$ is required to avoid double counting states. To compute this width numerically, we employ Eq. (42) and write

$$
\begin{aligned}
\frac{1}{2} \sum_{\vec{k}>0, \vec{l}>0} & =\frac{1}{2}\left(\frac{1}{2}\right)^{2} \int d m_{k}^{2} d m_{l}^{2} \rho_{\delta}\left(m_{k}^{2}\right) \rho_{\delta}\left(m_{l}^{2}\right) \\
& =\frac{1}{2}\left(\frac{1}{2}\right)^{2} \rho_{\delta}^{2}\left(m_{h}^{2}\right) m_{h}^{4} \int d x(\sqrt{x})^{\delta-2} \int d y(\sqrt{y})^{\delta-2} \\
& =\frac{1}{2}\left(\frac{1}{2}\right)^{2} \rho_{\delta}^{2}\left(m_{h}^{2}\right) m_{h}^{4} \int_{0}^{1} d x x^{\frac{\delta}{2}-1} \int_{0}^{1} d z(1-\sqrt{x})^{2}\left[(1-\sqrt{x})^{2} z\right]^{\frac{\delta}{2}-1}
\end{aligned}
$$

where we have defined

$$
x \equiv \frac{m_{l}^{2}}{m_{h}^{2}}, \quad y \equiv(1-\sqrt{x})^{2} z \equiv \frac{m_{k}^{2}}{m_{h}^{2}}
$$


and used the definition of the density, $\rho_{\delta}$, given in Eq. (11). The integration limits derive from the presence of the $\lambda$ kinematic phase space factor, which reduces to

$$
\lambda\left(m_{h}^{2}, m_{\vec{k}}^{2}, m_{\vec{l}}^{2}\right)=m_{h}^{2} \lambda(1, x, y)=m_{h}^{2}(1-\sqrt{x}) \sqrt{1-z}[1+x(1-z)+2 \sqrt{x}(1+z)-z]^{1 / 2},
$$

from which one immediately sees that phase space runs out at $x=1$ or $z=1$. In terms of the $x$ and $z$ variables, we have

$$
g_{\vec{k} \vec{l}}=\frac{1}{v m_{h}^{2}}\left\{\frac{1}{\xi}-2\left(1-\frac{1}{6 \xi}\right)\left(x+(1-\sqrt{x})^{2} z\right)\right\} .
$$

The final expression for $\Gamma(h \rightarrow$ graviscalar pairs $)$ can be written in terms of the integral

$$
\begin{aligned}
I= & \frac{1}{4} \int_{0}^{1} d x \int_{0}^{1} d z(1-\sqrt{x})^{\delta+1} \sqrt{1-z}[1+x(1-z)+2 \sqrt{x}(1+z)-z]^{1 / 2} x^{\delta / 2-1} z^{\delta / 2-1} \\
& \times\left|\frac{1}{\xi}-2\left(1-\frac{1}{6 \xi}\right)\left(x+(1-\sqrt{x})^{2} z\right)\right|^{2} .
\end{aligned}
$$

$I$ behaves as $1 / \xi^{2}$ at small $\xi$, reaches a minimum near $\xi=1.5$ due to the cancellations implicit in $g_{\vec{k} \vec{l}}$, and ultimately asymptotes (quite slowly) to a constant value of $I \rightarrow 0.011$ for $\delta=2(I \rightarrow 0.00024$ for $\delta=4)$ at $\xi \rightarrow \infty$. We plot $\xi^{2} I$ as a function of $\xi$ for the $\delta=2$ and $\delta=4$ cases in Fig. 3. Clearly, $I$ decreases rapidly as $\delta$ increases. As a result, $\Gamma(h \rightarrow$ graviscalar pairs $)$ is only significant compared to $\Gamma(h \rightarrow$ graviscalar $)$ if $\delta \leq 4$.
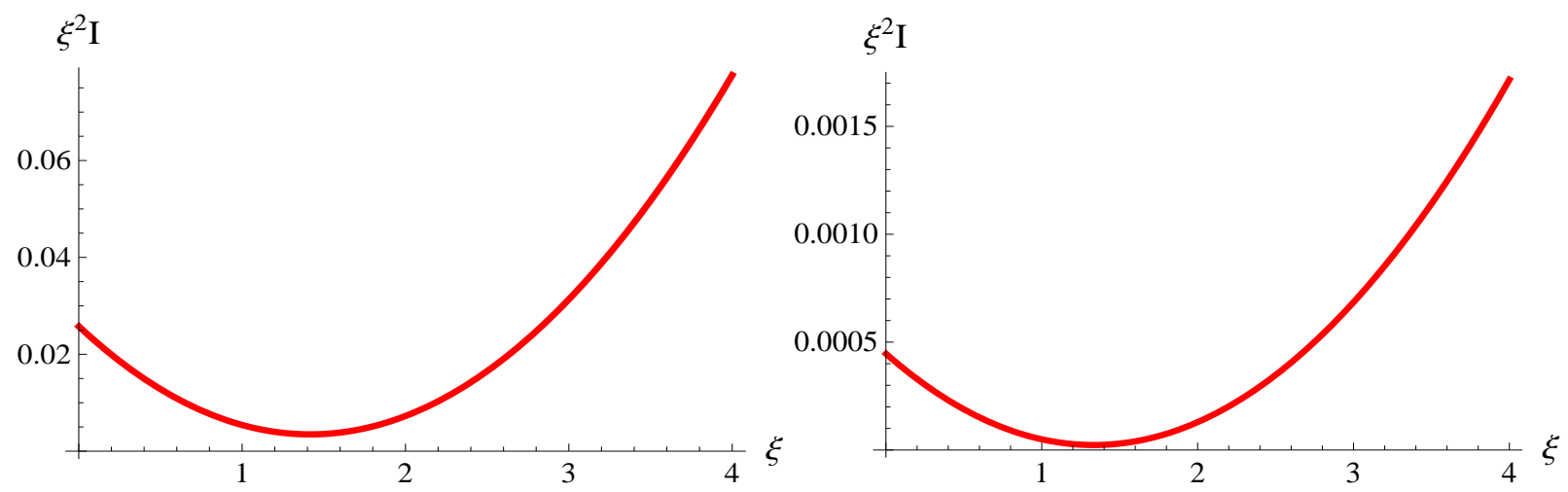

FIG. 3: We plot $\xi^{2} I$ as a function of $\xi$ for the cases of $\delta=2$ and $\delta=4$.

In terms of $I$, we find

$$
\begin{aligned}
\Gamma(h \rightarrow \text { graviscalar pairs }) & =\frac{1}{32 \pi} \frac{m_{h}^{2 \delta-5} \epsilon^{4}}{v^{2}} \frac{M_{P}^{4}}{M_{D}^{4+2 \delta}}\left[\frac{\pi^{\delta / 2}}{\Gamma(\delta / 2)}\right]^{2} I \\
& =\frac{18}{\pi} \frac{m_{h}^{3+2 \delta} v^{2}}{M_{D}^{4+2 \delta}} \xi^{4}\left(\frac{\delta-1}{\delta+2}\right)^{2}\left[\frac{\pi^{\delta / 2}}{\Gamma(\delta / 2)}\right]^{2} I .
\end{aligned}
$$

This is to be compared to the direct mixing invisible width, which in terms of $\epsilon$ takes the form:

$$
\Gamma(h \rightarrow \text { graviscalar })=\frac{\pi}{2} m_{h}^{\delta-3} \epsilon^{2} \frac{M_{P}^{2}}{M_{D}^{2+\delta}} \frac{\pi^{\delta / 2}}{\Gamma(\delta / 2)} .
$$

We obtain

$$
\begin{aligned}
\frac{\Gamma(h \rightarrow \text { graviscalar pairs })}{\Gamma(h \rightarrow \text { graviscalar })} & =\frac{1}{16 \pi^{2}} \frac{m_{h}^{\delta-2} \epsilon^{2}}{v^{2}} \frac{M_{P}^{2}}{M_{D}^{2+\delta}} \frac{\pi^{\delta / 2}}{\Gamma(\delta / 2)} I \\
& =\frac{3(\delta-1)}{2 \pi^{2}(\delta+2)} \xi^{2}\left(\frac{m_{h}}{M_{D}}\right)^{2+\delta} \frac{\pi^{\delta / 2}}{\Gamma(\delta / 2)} I .
\end{aligned}
$$


From this result, we immediately see that unless $m_{h}$ is comparable to or larger than $M_{D}$ the pair invisible width will be much smaller than the mixing invisible width unless $\xi^{2}$ is large enough, $\xi \sim 10$, to overcome the numerically small value of $I \sim 0.011$ at large $\xi$. However, for such large $\xi$ the mixing invisible width is typically huge. At small $\xi$, since $\xi^{2} I$ approaches a constant value so does $\Gamma(h \rightarrow$ graviscalar pairs $) / \Gamma(h \rightarrow$ graviscalar $)$.

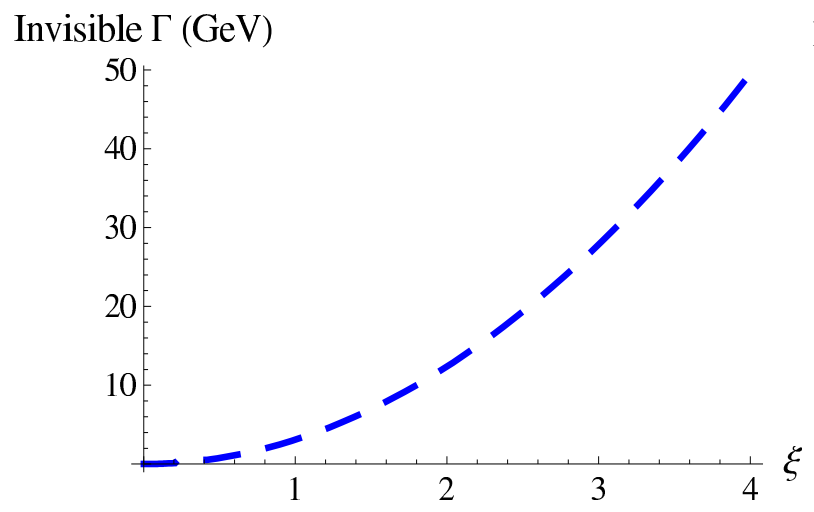

$\Gamma$ (graviscalar pairs) $/ \Gamma$ (graviscalar)

FIG. 4: We display $\Gamma_{i n v}=\Gamma(h \rightarrow$ graviscalar $)$ and $\Gamma(h \rightarrow$ graviscalar pairs $) / \Gamma(h \rightarrow$ graviscalar $)$ for $\delta=2, M_{D}=1 \mathrm{TeV}$ and $m_{h}=120 \mathrm{GeV}$.

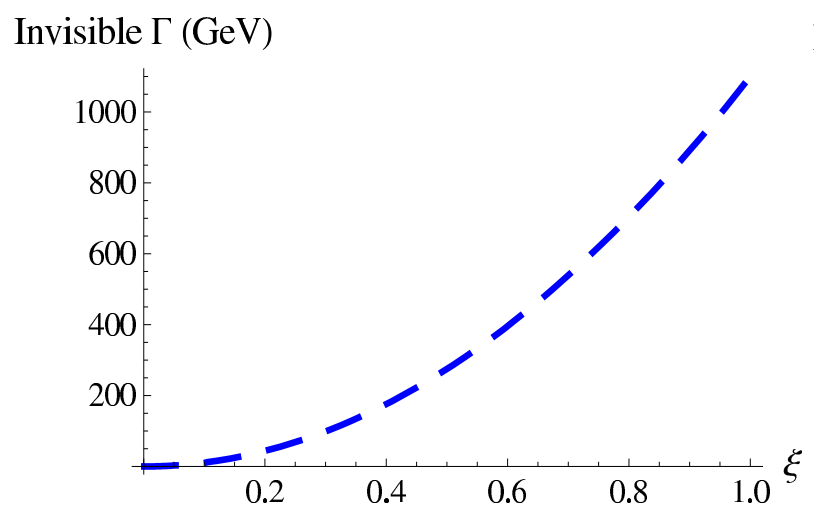

$\Gamma$ (graviscalar pairs) $/ \Gamma$ (graviscalar)

FIG. 5: We display $\Gamma_{i n v}=\Gamma(h \rightarrow$ graviscalar $)$ and $\Gamma(h \rightarrow$ graviscalar pairs $) / \Gamma(h \rightarrow$ graviscalar $)$ for $\delta=2, M_{D}=1 \mathrm{TeV}$ and $m_{h}=850 \mathrm{GeV}$.

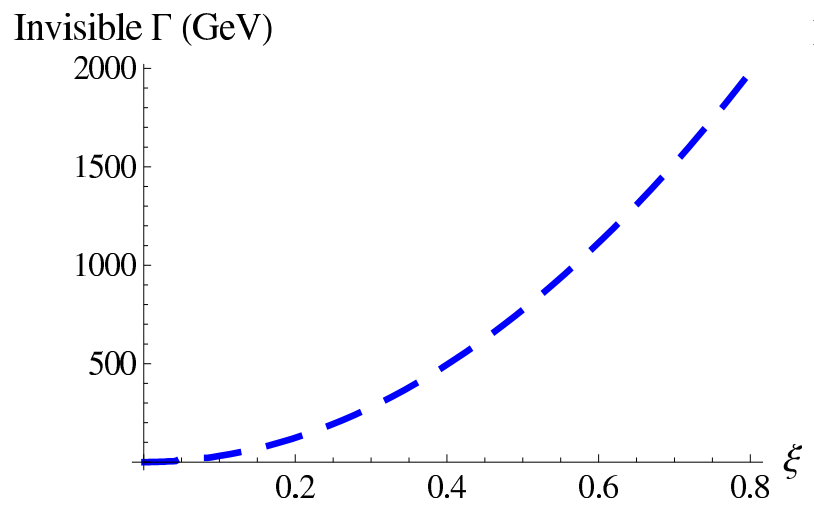

$\Gamma($ graviscalar pairs $) / \Gamma($ graviscalar $)$

FIG. 6: We display $\Gamma_{i n v}=\Gamma(h \rightarrow$ graviscalar $)$ and $\Gamma(h \rightarrow$ graviscalar pairs $) / \Gamma(h \rightarrow$ graviscalar $)$ for $\delta=2, M_{D}=1$ TeV and $m_{h}=1200 \mathrm{GeV}$. 

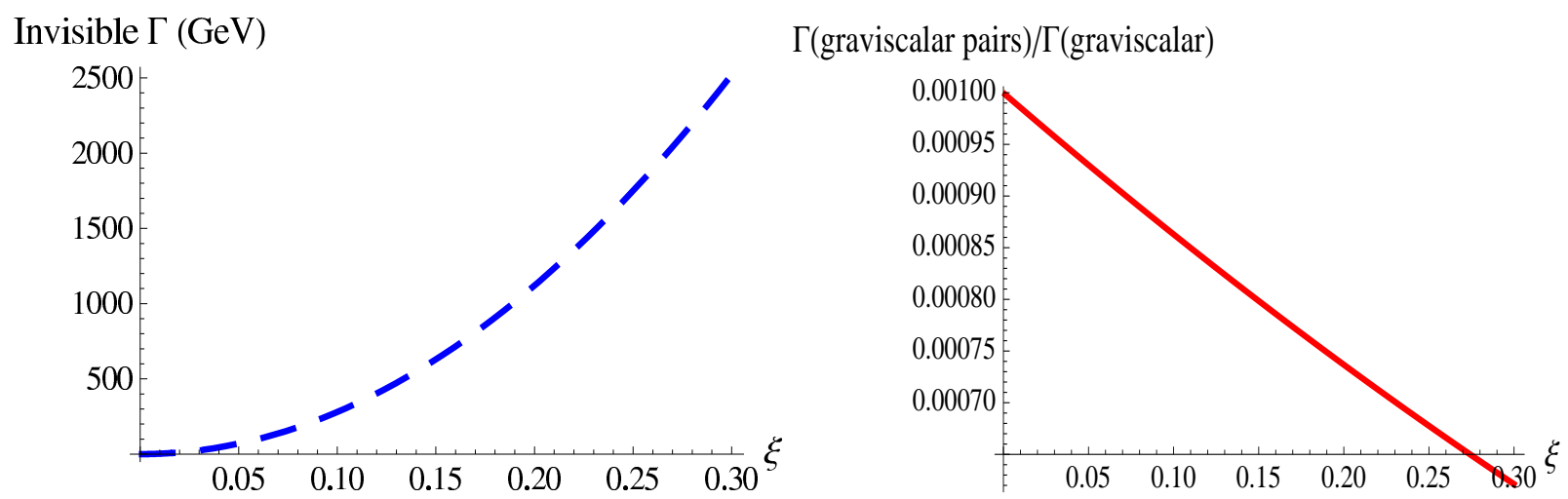

FIG. 7: We display $\Gamma_{i n v}=\Gamma(h \rightarrow$ graviscalar $)$ and $\Gamma(h \rightarrow$ graviscalar pairs $) / \Gamma(h \rightarrow$ graviscalar $)$ for $\delta=4, M_{D}=1 \mathrm{TeV}$ and $m_{h}=1200 \mathrm{GeV}$.

To illustrate, we display in Figs. 4, 5 and 6 the mixing invisible width $\Gamma(h \rightarrow$ graviscalar $)$ and the ratio $\Gamma(h \rightarrow$ graviscalar pairs $) / \Gamma(h \rightarrow$ graviscalar $)$ as a function of $\xi$ for the cases of $\delta=2, M_{D}=1 \mathrm{TeV}$ (the approximate lower limit for $\delta=2$ from Tevatron data) and a selection of Higgs masses: $m_{h}=120 \mathrm{GeV}$, $m_{h}=850 \mathrm{GeV}$ and $m_{h}=1200 \mathrm{GeV}$. One finds that the graviscalar-pair to graviscalar-mixing width ratio is very small for the light Higgs case and only as large as a percent even for $m_{h}$ somewhat above $1 \mathrm{TeV}$, a range of $m_{h}$ that becomes questionable from the point of view of unitarity for $W W \rightarrow W W$ scattering. For $\delta=4$, the ratio is even smaller as apparent from the example of $\delta=4, M_{D}=1 \mathrm{TeV}$ and $m_{h}=1200 \mathrm{GeV}$ plotted in Fig. 7 . In the above plots, one should presumably not take seriously the regions at larger $\xi$ in the $m_{h}=850 \mathrm{GeV}$ and $1200 \mathrm{GeV}$ cases for which $\Gamma_{\text {inv }}$ becomes substantially larger than $m_{h}$.

\section{CONCLUSIONS}

We computed the amplitude for a process such as $W W \rightarrow W W$ in the presence of Higgs-graviscalar mixing. Using a direct Feynman diagram approach we performed the calculation in two different bases: (a) the non-massdiagonal (Lagrangian) basis and (b) the diagonalized mass basis. Of course, identical results were obtained but the comparison provides a few pedagogical and intuitive insights. Ignoring small corrections from wave-function renormalization, the resulting amplitude is equivalent to that for exchange of a single effective Higgs state with SM coupling to $W W$ but effective width given by $\Gamma_{h}^{S M}+\Gamma_{\text {inv }}$, where $\Gamma_{h}^{S M}$ is the Higgs width as computed in the standard model and (up to the factor of 2 correction that we found) $\Gamma_{\text {inv }}$ is the width obtained via the original technique of Ref. [7]. In particular, one would not observe a sum of Breit-Wigners, one of width $\Gamma_{h}^{S M}$ overlaying a superposition of many much narrower resonances. Integration over the full resonance will yield a $W W \rightarrow W W$ cross section that is suppressed compared to the SM result by the factor $1 /(1+R)$, where $R=\Gamma_{\text {inv }} / \Gamma_{h}^{S M}$. Of course, the width and total cross section for any process mediated by Higgs exchange will be affected in exactly the same way as is $W W \rightarrow W W$. For example, for any choices of $\delta, \xi$ and $M_{D}$ such that $\Gamma_{h}^{S M}+\Gamma_{\text {inv }} \gtrsim 2 \mathrm{GeV}$ it will be possible to directly measure this net width in the process $g g \rightarrow H i g g s \rightarrow Z Z \rightarrow 4 \ell$ by looking at the peak shape in $M_{4 \ell}$.

Using the Feynman diagram technique we were also able to perform a direct computation of the invisible width from $W W \rightarrow h \rightarrow \sum_{\vec{n}, \vec{k}} s_{\vec{n}} s_{\vec{k}}$ on resonance pair production of graviscalars. We found that this width was typically much smaller than the invisible width from Higgs-graviscalar mixing unless $m_{h}>M_{D}$ ( $M_{D}$ being the effective $4+\delta$ dimensional Planck scale). However, the $m_{h}>M_{D}$ region of parameter space (a) is problematical from the point of view of $W W \rightarrow W W$ unitarity given that $M_{D}>1 \mathrm{TeV}$ and (b) is such that many other corrections to the invisible width are possibly present. Nonetheless, for $m_{h}>M_{D}$ including the pair width would be necessary for precision comparison between theory and experiment. 


\section{ACKNOWLEDGMENTS}

D. Dominici was partially supported by MIUR under the contract PRIN-2006020509. JFG is supported by the U.S. Department of Energy under grant No. DE-FG03-91ER40674. JFG would also like to thank the University of Florence, the INFN, the Aspen Center for Physics and the Kavli Institute for Theoretical Physics for support during various parts of this project.

\section{APPENDIX A: FEYNMAN DIAGRAM DERIVATION OF THE INVISIBLE WIDTH AND FULL $W W \rightarrow W W$ SCATTERING AMPLITUDE FORM}

To begin with, it is useful to understand how the calculation should be done using the example of a small number of states. We illustrate using 3 states. The three states are the $h$ (Higgs) with mass-squared $\mu^{2}$, a graviscalar $s_{1}$ with mass $m_{1}$ and a second graviscalar $s_{2}$ with mass $m_{2}$.

Since the $h$ is the only one of the states with couplings to $W W$ and $f \bar{f}$ (that are not suppressed by $1 / M_{P}$ ) it is the only state with a substantial imaginary component, $i \frac{1}{2} \mu \Gamma_{h}$.

The relevant mass-squared matrix is (using Eq. (14))

$$
\frac{1}{2} M^{2} \equiv \frac{1}{2}\left(\begin{array}{ccc}
\mu^{2}-i \mu \Gamma_{h} & -\epsilon & -\epsilon \\
-\epsilon & m_{1}^{2} & 0 \\
-\epsilon & 0 & m_{2}^{2}
\end{array}\right)
$$

This $3 \times 3$ matrix is a complex symmetric matrix, that is not hermitian. It can be diagonalized by an orthogonal transformation (given that the eigenvalues are distinct). To sufficient order, the eigenvalues are

$$
\mu^{2}-i \rho+\epsilon^{2} \frac{1}{\mu^{2}-i \rho-m_{1}^{2}}+\epsilon^{2} \frac{1}{\mu^{2}-i \rho-m_{2}^{2}}, \quad m_{1}^{2}+\frac{\epsilon^{2}}{m_{1}^{2}-\mu^{2}+i \rho}, \quad m_{2}^{2}+\frac{\epsilon^{2}}{m_{2}^{2}-\mu^{2}+i \rho},
$$

where we have written $\rho \equiv \mu \Gamma_{h}$. The eigenvectors of the $3 \times 3$ matrix are (dropping terms of $\mathcal{O}\left(\epsilon^{3}\right)$ and higher)

$$
\begin{aligned}
& w_{1}=\left(\begin{array}{c}
1-\frac{1}{2} \epsilon^{2}\left(\frac{1}{\left(m_{1}^{2}-\mu^{2}+i \rho\right)^{2}}+\frac{1}{\left(m_{2}^{2}-\mu^{2}+i \rho\right)^{2}}\right) \\
\frac{-m_{1}^{2}-i \rho}{\mu^{2}-m_{2}^{2}-i \rho}
\end{array}\right) \\
& w_{2}=\left(\begin{array}{c}
\frac{\epsilon}{\mu^{2}-i \rho-m_{1}^{2}} \\
1-\frac{1}{2} \frac{\epsilon^{2}}{\left(m_{1}^{2}-\mu^{2}+i \rho\right)^{2}} \\
-\frac{1}{2} \frac{\epsilon^{2}}{\left(m_{1}^{2}-\mu^{2}-i \rho\right)\left(m_{2}^{2}-\mu^{2}-i \rho\right)}
\end{array}\right) \\
& w_{3}=\left(\begin{array}{c}
\frac{\epsilon}{\mu^{2}-i \rho-m_{2}^{2}} \\
-\frac{1}{2} \frac{\epsilon^{2}}{\left(m_{2}^{2}-\mu^{2}-i \rho\right)\left(m_{1}^{2}-\mu^{2}-i \rho\right)} \\
1-\frac{1}{2} \frac{\epsilon^{2}}{\left(m_{2}^{2}-\mu^{2}+i \rho\right)^{2}}
\end{array}\right) .
\end{aligned}
$$

These form a normalized orthogonal basis in the sense that $w_{i}^{T} w_{i}=\delta_{i j}$ for $i, j=1,2,3$ and not $w_{i}^{\dagger} w_{j}=\delta_{i j}$. The matrix which diagonalizes the mass-squared matrix is built as

$$
T=\left\{w_{1}, w_{2}, w_{3}\right\}
$$

and one can check that (to order $\epsilon^{2}$ )

$$
T^{-1} M^{2} T=M_{D}^{2}
$$

where $M_{D}^{2}$ is the diagonal matrix containing the eigenvalues of Eq. (A2). Also, to order $\epsilon^{2}, T^{-1}$ coincides with the transpose of $T$. Therefore the relation between $\phi=\left\{h, s_{1}, s_{2}\right\}$ and the mass eigenstates $\phi^{\prime}=\left\{h^{\prime}, s_{1}^{\prime}, s_{2}^{\prime}\right\}$ is given by

$$
\phi^{\prime}=T^{T} \phi \quad \phi=T \phi^{\prime} .
$$

from which we obtain

$$
\begin{aligned}
h & =w_{1}(1) h^{\prime}+w_{2}(1) s_{1}^{\prime}+w_{3}(1) s_{2}^{\prime} \\
& =\left[1-\frac{1}{2} \epsilon^{2}\left(\frac{1}{\left(m_{1}^{2}-\mu^{2}+i \rho\right)^{2}}+\frac{1}{\left(m_{2}^{2}-\mu^{2}+i \rho\right)^{2}}\right)\right] h^{\prime}+\frac{\epsilon}{\mu^{2}-i \rho-m_{1}^{2}} s_{1}^{\prime}+\frac{\epsilon}{\mu^{2}-i \rho-m_{2}^{2}} s_{2}^{\prime}
\end{aligned}
$$


where $w_{i}(1)$ is the first component of the vector $w_{i}$. Similarly, we have

$$
\begin{aligned}
s_{1} & =w_{1}(2) h^{\prime}+w_{2}(2) s_{1}^{\prime}+w_{3}(2) s_{2}^{\prime} \\
& \simeq \frac{-\epsilon}{\mu^{2}-m_{1}^{2}-i \rho} h^{\prime}+\left(1-\frac{1}{2} \frac{\epsilon^{2}}{\left(m_{1}^{2}-\mu^{2}+i \rho\right)^{2}}\right) s_{1}^{\prime}-\frac{1}{2} \frac{\epsilon^{2}}{\left(m_{2}^{2}-\mu^{2}-i \rho\right)\left(m_{1}^{2}-\mu^{2}-i \rho\right)} s_{2}^{\prime},
\end{aligned}
$$

and similarly for $s_{2}$.

Of course, since the transformation is orthogonal the kinetic terms for the original $h, s_{1}$ and $s_{2}$ states transform into

$$
\frac{1}{2}\left(\partial_{\mu} h^{\prime} \partial^{\mu} h^{\prime}+\partial_{\mu} s_{1}^{\prime} \partial^{\mu} s_{1}^{\prime}+\partial_{\mu} s_{2}^{\prime} \partial^{\mu} s_{2}^{\prime}\right)
$$

where we recall that the fields $h, s_{1}, s_{2}$ were real while these new $h^{\prime}, s_{1}^{\prime}, s_{2}^{\prime}$ now have (small) complex components.

For later use, we will want the $W W$ coupling of each of the $3 h^{\prime}, s_{1}^{\prime}, s_{2}^{\prime}$ states. This comes entirely from the $h$ part of each state giving (relative to the SM coupling $g_{W W h}$ )

$$
\begin{aligned}
g_{W W h^{\prime}} & =1-\frac{1}{2} \epsilon^{2}\left[\frac{1}{\left(m_{1}^{2}-\mu^{2}+i \rho\right)^{2}}+\frac{1}{\left(m_{2}^{2}-\mu^{2}+i \rho\right)^{2}}\right] \\
g_{W W s_{1}^{\prime}} & =\frac{\epsilon}{\mu^{2}-i \rho-m_{1}^{2}} \\
g_{W W s_{2}^{\prime}} & =\frac{\epsilon}{\mu^{2}-i \rho-m_{2}^{2}} .
\end{aligned}
$$

These are converted to Feynman rules for the vertices as usual. Note, that the couplings are complex; this will be important in what follows.

From the above, the generalization to many $s_{i}$ states is apparent. For the $h^{\prime}$ mass-squared we find

$$
\begin{aligned}
m_{h^{\prime}}^{2} & =\mu^{2}-i \rho+\epsilon^{2} \sum_{\vec{n}>0} \frac{1}{\mu^{2}-i \rho-m_{\vec{n}}^{2}} \\
& =\mu^{2}-i \rho+\epsilon^{2} \sum_{\vec{n}>0} \frac{\mu^{2}-m_{\vec{n}}^{2}+i \rho}{\left(\mu^{2}-m_{\vec{n}}^{2}\right)^{2}+\rho^{2}} \\
& \simeq \mu^{2}\left(1+\frac{\epsilon^{2}}{\mu^{2}} \Re\left[\sum_{\vec{n}>0} \frac{1}{\mu^{2}-i \rho-m_{\vec{n}}^{2}}\right]\right)-i\left(\rho-\epsilon^{2} \sum_{\vec{n}>0} \pi \delta\left(\mu^{2}-m_{\vec{n}}^{2}\right)\right) \\
& \sim \mu^{2}-i\left(\rho-\rho_{i n v}\right)
\end{aligned}
$$

where we have neglected the $\Re$ term (which is of order $\left.\mu^{4} / M_{D}^{4}\right)$. Note that in the $h^{\prime}$ propagator, $i /\left(p^{2}-m_{h^{\prime}}^{2}\right)$, the invisible width comes in with what appears to be a "wrong" sign. However, as we will see in the following, in a typical physical amplitude, one must sum over both $h^{\prime}$ and $s_{\vec{n}}^{\prime}$ exchanges. The sum produces an effective propagator of form $i /\left[p^{2}-\mu^{2}+i\left(\rho+\rho_{\text {inv }}\right)\right]$. Below we show this explicitly for $W W \rightarrow W W$ scattering.

First, however, we must give expressions for the $W W$ couplings to the $h^{\prime}$ and $s_{\vec{n}}^{\prime}$ states. We have

$$
g_{W W h^{\prime}}=1-\frac{1}{2} \sum_{\vec{n}>0} \epsilon^{2} \frac{1}{\left(m_{\vec{n}}^{2}-\mu^{2}+i \rho\right)^{2}}, \quad g_{W W s_{\vec{n}}^{\prime}}=\frac{\epsilon}{\mu^{2}-i \rho-m_{\vec{n}}^{2}} .
$$

The $W W \rightarrow W W$ amplitude is then the sum $W W \rightarrow h^{\prime} \rightarrow W W+\sum_{\vec{n}>0} W W \rightarrow s_{\vec{n}}^{\prime} \rightarrow W W$ and takes the form:

$$
\begin{aligned}
& \mathcal{A}_{W W \rightarrow h^{\prime} \rightarrow W W}+\sum_{\vec{n}>0} \mathcal{A}_{W W \rightarrow s_{\vec{n}}^{\prime} \rightarrow W W} \\
\sim & \frac{i}{p^{2}-\mu^{2}+i \rho+\sum_{\vec{n}>0} \frac{\epsilon^{2}}{m_{\vec{n}}^{2}-\mu^{2}+i \rho}}\left(1-\frac{1}{2} \sum_{\vec{n}>0} \frac{\epsilon^{2}}{\left(m_{\vec{n}}^{2}-\mu^{2}+i \rho\right)^{2}}\right)^{2}+\sum_{\vec{n}>0} \frac{i}{p^{2}-m_{\vec{n}}^{2}-\frac{\epsilon^{2}}{m_{\vec{n}}^{2}-\mu^{2}+i \rho}}\left(\frac{-\epsilon}{m_{\vec{n}}^{2}-\mu^{2}+i \rho}\right)^{2} \\
\sim & \frac{i}{p^{2}-\mu^{2}+i \rho+\sum_{\vec{n}>0} \frac{\epsilon^{2}}{m_{\vec{n}}^{2}-\mu^{2}+i \rho}}\left(1-\sum_{\vec{n}>0} \frac{\epsilon^{2}}{\left(m_{\vec{n}}^{2}-\mu^{2}+i \rho\right)^{2}}\right)+\sum_{\vec{n}>0} \frac{\epsilon^{2}}{p^{2}-m_{\vec{n}}^{2}-\frac{\epsilon^{2}}{m_{\vec{n}}^{2}-\mu^{2}+i \rho}} \frac{\left.m_{\vec{n}}^{2}-\mu^{2}+i \rho\right)^{2}}{}
\end{aligned}
$$




$$
\begin{aligned}
& \sim \frac{i}{p^{2}-\mu^{2}+i \rho+\sum_{\vec{n}>0} \frac{\epsilon^{2}}{m_{\vec{n}}^{2}-\mu^{2}+i \rho}}\left[1-\sum_{\vec{n}>0} \frac{\epsilon^{2}}{\left(m_{\vec{n}}^{2}-\mu^{2}+i \rho\right)^{2}}+\sum_{\vec{n}>0} \frac{p^{2}-\mu^{2}+i \rho}{p^{2}-m_{\vec{n}}^{2}} \frac{\epsilon^{2}}{\left(m_{\vec{n}}^{2}-\mu^{2}+i \rho\right)^{2}}\right] \\
& \left.\left.\sim \frac{i}{p^{2}-\mu^{2}+i \rho+\sum_{\vec{n}>0} \frac{\epsilon^{2}}{m_{\vec{n}}^{2}-\mu^{2}+i \rho}}\right] 1+\sum_{\vec{n}>0} \frac{\epsilon^{2}}{\left(m_{\vec{n}}^{2}-\mu^{2}+i \rho\right)^{2}} \frac{\epsilon^{2}}{p^{2}-m_{\vec{n}}^{2}}\right] \\
& \sim \frac{i}{p^{2}-\mu^{2}+i \rho+\sum_{\vec{n}>0} \frac{\epsilon^{2}}{m_{\vec{n}}^{2}-\mu^{2}+i \rho}}\left[1+\sum_{\vec{n}>0} \frac{\left.\left.i p_{\vec{n}}^{2}\right)\right)}{\left(m_{\vec{n}}^{2}-\mu^{2}+i \rho\right)\left(p^{2}-m_{\vec{n}}^{2}\right)}\right] \\
& \sim \frac{\left.p^{2}-\mu^{2}+i \rho+\sum_{\vec{n}>0} \frac{\epsilon^{2}}{m_{\vec{n}}^{2}-\mu^{2}+i \rho}\right]\left[1-\sum_{\vec{n}>0} \frac{\epsilon^{2}}{\left(m_{\vec{n}}^{2}-\mu^{2}+i \rho\right)\left(p^{2}-m_{\vec{n}}^{2}\right)}\right]}{p^{2}-\mu^{2}+i \rho+\epsilon^{2} \sum_{\vec{n}>0}\left(\frac{1}{m_{\vec{n}}^{2}-\mu^{2}+i \rho}-\frac{p^{2}-\mu^{2}+i \rho}{\left(m_{\vec{n}}^{2}-\mu^{2}+i \rho\right)\left(p^{2}-m_{\vec{n}}^{2}\right)}\right)} \\
& \sim \frac{i}{p^{2}-\mu^{2}+i \rho+\epsilon^{2} \sum_{\vec{n}>0}\left(\frac{1}{m_{\vec{n}}^{2}-\mu^{2}+i \rho}-\frac{1}{p^{2}-m_{\vec{n}}^{2}}-\frac{1}{m_{\vec{n}}^{2}-\mu^{2}+i \rho}\right)} \\
& \sim \frac{i}{p^{2}-\mu^{2}+i \rho-\sum_{\vec{n}>0} \frac{\epsilon^{2}}{p^{2}-m_{\vec{n}}^{2}}} \\
& \sim \frac{i}{p^{2}-\mu^{2}+i \rho-\frac{1}{2} \int d m^{2} \rho_{\delta}\left(m^{2}\right) \frac{\epsilon^{2}}{p^{2}-m^{2}+i \epsilon^{\prime}}} \\
& \sim \frac{i \rho+F\left(p^{2}\right)+i G\left(p^{2}\right)}{p^{2}+i}
\end{aligned}
$$

where

$$
F\left(p^{2}\right)=-\epsilon^{2} P \frac{1}{2}\left[\int \frac{d m^{2} \rho_{\delta}\left(m^{2}\right)}{p^{2}-m^{2}}\right], \quad G\left(p^{2}\right)=\frac{1}{2} \pi \epsilon^{2} \rho_{\delta}\left(p^{2}\right) .
$$

The most critical step in the above is the transition from line 6 to line 7 of Eq. A13 in which we presume the higher order terms of order $\epsilon^{4}$ and so forth organize into the correct geometric series (as they did in the approach of Ref. [7] $)$. We next write $F\left(p^{2}\right)=F\left(m_{h}^{2}\right)+\left(p^{2}-m_{h}^{2}\right) F^{\prime}\left(m_{h}^{2}\right)+\ldots$, where $m_{h}^{2}-\mu^{2}+F\left(m_{h}^{2}\right)=0$ and drop the .... We also approximate $G\left(p^{2}\right)=G\left(m_{h}^{2}\right)$ and use the result of Eq. (9) to obtain

$$
\mathcal{A}_{W W \rightarrow W W} \sim \frac{i}{\left(p^{2}-m_{h}^{2}\right)\left[1+F^{\prime}\left(m_{h}^{2}\right)\right]+i m_{h}\left(\Gamma_{h}+\Gamma_{i n v}\right)} .
$$

The result above shows that the $W W \rightarrow W W$ scattering amplitude is indeed equivalent to that for a single Higgs exchange with total width given by $\Gamma_{h}^{S M}+\Gamma_{\text {inv }}$, aside from wave-function renormalization associated with the graviscalar mixing. However, the wave-function renormalization correction is small since $F^{\prime} \sim \mathcal{O}\left(m_{h}^{4} / M_{D}^{4}\right)$ is very small for $m_{h} \ll M_{D}$, as required for the model to be fully trustworthy.

If we now take the absolute square of this form and integrate over $p^{2}$, we obtain

$$
\begin{aligned}
\int d p^{2}\left|\frac{i}{\left(p^{2}-m_{h}^{2}\right)\left[1+F^{\prime}\left(m_{h}^{2}\right)\right]+i \mu\left(\Gamma_{h}+\Gamma_{i n v}\right)}\right|^{2} & =\int d p^{2} \frac{1}{\left(p^{2}-m_{h}^{2}\right)^{2}\left[1+F^{\prime}\right]^{2}+m_{h}^{2}\left(\Gamma_{h}+\Gamma_{i n v}\right)^{2}} \\
& \sim \frac{1}{1+F^{\prime}} \frac{\pi}{m_{h}\left(\Gamma_{h}+\Gamma_{i n v}\right)},
\end{aligned}
$$

which is to be compared to the result we would have obtained in the absence of graviscalars, which is $\sim \frac{\pi}{m_{h} \Gamma_{h}}$. Eq. (A16) shows that (neglecting wave-function renormalization) the integral over $p^{2}=s_{W W}$ of the $W W \rightarrow W W$ scattering amplitude gives the $W W$ partial width (which has been implicitly set to unity for this discussion) divided by the total width including the graviscalar mixing contribution.

The above discussion neglects a small correction to the $h^{\prime}$ and $s_{\vec{n}}^{\prime}$ couplings deriving from the $s_{\vec{n}}$ couplings. These take the form (in the general case):

$$
g_{W W s_{\vec{n}}}=\frac{\epsilon(1-6 \xi) m_{W}^{2}}{3 \xi v \mu^{2}}=g_{W W h} \frac{\epsilon(1-6 \xi) m_{W}}{3 \xi g v \mu^{2}}=g_{W W h} \frac{\epsilon(1-6 \xi)}{6 \xi \mu^{2}}
$$


where we were careful to rewrite $T_{\mu}^{\mu}=-m_{V}^{2} \vec{V}_{\mu} \cdot \vec{V}^{\mu}=-m_{V}^{2}\left(2 W_{\mu}^{+} W^{\mu-}+W_{\mu}^{3} W^{3 \mu}\right)$, giving rise to an extra factor of 2 for $W^{+} W^{-}$couplings. This result actually applies to all types of couplings: in particular, we also have

$$
g_{f \bar{f} s_{\vec{n}}}=-\frac{\epsilon(1-6 \xi) m_{f}}{6 \xi v \mu^{2}}=g_{f \bar{f} h} \frac{\epsilon(1-6 \xi)}{6 \xi \mu^{2}} .
$$

We define the common $W W$ and $f \bar{f}$ ratio as

$$
\gamma \equiv \frac{\epsilon(1-6 \xi)}{6 \xi \mu^{2}}
$$

We note that the $(1-6 \xi)$ factor appears in all the graviscalar couplings.

In addition, it is useful to define

$$
r_{\vec{n}} \equiv \frac{\gamma}{\epsilon}\left(m_{\vec{n}}^{2}-\mu^{2}+i \rho\right)
$$

Then, the full $W W h^{\prime}$ and $W W s^{\prime}$ couplings take the form

$$
\begin{aligned}
g_{W W h^{\prime}} & =\left(1-\frac{1}{2} \sum_{\vec{n}>0} \epsilon^{2} \frac{1}{\left(m_{\vec{n}}^{2}-\mu^{2}+i \rho\right)^{2}}\right) g_{W W h}+\sum_{\vec{n}>0}\left(\frac{-\epsilon}{\mu^{2}-m_{\vec{n}}^{2}-i \rho}\right) g_{W W s_{\vec{n}}} \\
& =\left(1-\frac{1}{2} \sum_{\vec{n}>0} \frac{\epsilon^{2}}{\left(m_{\vec{n}}^{2}-\mu^{2}+i \rho\right)}\left[1-2 r_{\vec{n}}\right]\right) g_{W W h}
\end{aligned}
$$

and, similarly,

$$
g_{W W s_{\vec{n}}^{\prime}}=\frac{\epsilon}{\mu^{2}-i \rho-m_{\vec{n}}^{2}} g_{W W h}+\left(1-\frac{1}{2} \frac{\epsilon^{2}}{\left(m_{\vec{n}}^{2}-\mu^{2}+i \rho\right)^{2}}\right) \gamma g_{W W h}=-\frac{\epsilon}{m_{\vec{n}}^{2}+i \rho-\mu^{2}}\left[1-r_{\vec{n}}\right] g_{W W h}
$$

where we dropped the $\epsilon^{2}$ term in the big parenthesis since $\gamma$ is already of order $\epsilon$. We can now correct the computation we did in Eq. A13). Following the same type of procedure we obtain (dropping the common $\left.g_{W W h}\right)$ :

$$
\begin{aligned}
& \mathcal{A}_{W W \rightarrow W W} \\
\sim & \frac{i}{p^{2}-\mu^{2}+i \rho+\sum_{\vec{n}>0} \frac{\epsilon^{2}}{m^{2}-\mu^{2}+i \rho}}\left(1-\frac{1}{2} \sum_{\vec{n}>0} \frac{\epsilon^{2}}{\left(m_{\vec{n}}^{2}-\mu^{2}+i \rho\right)^{2}}\left[1-2 r_{\vec{n}}\right]\right)^{2}+\sum_{\vec{n}>0} \frac{i}{p^{2}-m_{\vec{n}}^{2}-\frac{\epsilon^{2}}{m_{\vec{n}}^{2}-\mu^{2}+i \rho}}\left(\frac{-\epsilon}{m_{\vec{n}}^{2}-\mu^{2}+i \rho}\left[1-r_{\vec{n}}\right]\right)^{2} \\
= & \frac{i}{p^{2}-\mu^{2}+i \rho-\sum_{\vec{n}>0} \frac{\epsilon^{2}}{p^{2}-m_{\vec{n}}^{2}}\left(1-\left(p^{2}-\mu^{2}+i \rho\right)\left[2 \frac{\gamma}{\epsilon}\right]+\left(p^{2}-\mu^{2}+i \rho\right)^{2}\left(\frac{\gamma}{\epsilon}\right)^{2}\right)} .
\end{aligned}
$$

In the on-shell approximation of $p^{2} \sim \mu^{2}$ the corrections to the terms we kept before are of order

$$
2 i \rho \frac{\gamma}{\epsilon}-\rho^{2}\left(\frac{\gamma}{\epsilon}\right)^{2} \sim 2 i \frac{\Gamma_{h}^{S M}}{\mu} \frac{1-6 \xi}{6 \xi}-\left(\frac{\Gamma_{h}^{S M}}{\mu}\right)^{2}\left(\frac{1-6 \xi}{6 \xi}\right)^{2}
$$

where we used $\rho=\mu \Gamma_{h}^{S M}$ (where $\mu$ is our short-hand notation for $m_{h}$ ). For a light Higgs, $\Gamma_{h}^{S M} / \mu$ is a very tiny number and this correction can be neglected. (Note that for small $\xi, \epsilon \propto \xi$ so that there is no actual $\xi$ singularity and the whole effect is simply very small.) Our numerical results presented in the main body of the paper neglected both this correction and the $F^{\prime}$ correction discussed earlier.

Let us finally conclude with the obvious generalizations of Eqs. (A7) and (A8):

$$
\begin{gathered}
h \sim N\left[h^{\prime}+\sum_{\vec{m}>0} \frac{\epsilon}{m_{h}^{2}-i \rho-m_{\vec{m}}^{2}} s_{\vec{m}}^{\prime}\right] \\
s_{n}=N_{\vec{n}}\left[s_{\vec{n}}^{\prime}-\frac{\epsilon}{m_{h}^{2}-i \rho-m_{\vec{n}}^{2}} h^{\prime}-\frac{1}{2} \frac{\epsilon^{2}}{\left(m_{\vec{n}}^{2}-m_{h}^{2}+i \rho\right)} \sum_{\vec{m} \neq \vec{n}, \vec{n}>0, \vec{m}>0} \frac{1}{m_{\vec{m}}^{2}-m_{h}^{2}+i \rho} s_{\vec{m}}^{\prime}\right]
\end{gathered}
$$


where $h$ and $s_{n}$ are the original fields before diagonalizing the Hamiltonian and

$$
\begin{gathered}
N \sim\left[1+\sum_{\vec{m}>0} \frac{\epsilon^{2}}{\left(m_{h}^{2}-i \rho-m_{\vec{m}}^{2}\right)^{2}}\right]^{-1 / 2} \\
N_{\vec{n}}=\left[1+\frac{\epsilon^{2}}{\left(m_{h}^{2}-i \rho-m_{\vec{n}}^{2}\right)^{2}}\right]^{-1 / 2} \sim 1+\mathcal{O}\left(\frac{1}{M_{P}^{2}}\right) .
\end{gathered}
$$

We emphasize again that Eqs. A25 and A26 yield a diagonal, canonically normalized form for the kinetic energy terms while the mass terms also take a diagonal form:

$$
\begin{aligned}
& -\frac{1}{2}\left[\left(m_{h}^{2}-i \rho\right) h^{2}+\sum_{\vec{n}>0} m_{\vec{n}}^{2} s_{n}^{2}-2 \epsilon h \sum_{\vec{n}>0} s_{n}\right] \\
= & -\frac{1}{2}\left[\left(m_{h}^{2}-i \rho+\epsilon^{2} \sum_{\vec{n}>0} \frac{1}{m_{h}^{2}-i \rho-m_{\vec{n}}^{2}}\right) h^{\prime 2}+\sum_{\vec{n}>0}\left(m_{\vec{n}}^{2}-\epsilon^{2} \frac{1}{m_{h}^{2}-i \rho-m_{\vec{n}}^{2}}\right) s_{\vec{n}}^{\prime 2}\right] \\
& +O\left(\epsilon^{3}\right),
\end{aligned}
$$

Let us study the $h^{\prime 2}$ mass squared. We have

$$
\begin{aligned}
m_{h^{\prime}}^{2} & =m_{h}^{2}-i \rho+\epsilon^{2} \sum_{\vec{n}>0} \frac{1}{m_{h}^{2}-i \rho-m_{\vec{n}}^{2}} \\
& =m_{h}^{2}-i \rho+\epsilon^{2} \sum_{\vec{n}>0} \frac{m_{h}^{2}-m_{\vec{n}}^{2}+i \rho}{\left(m_{h}^{2}-m_{\vec{n}}^{2}\right)^{2}+\rho^{2}} \\
& \simeq m_{h}^{2}\left(1+\frac{\epsilon^{2}}{m_{h}^{2}} \Re\left[\sum_{\vec{n}>0} \frac{1}{m_{h}^{2}-i \rho-m_{\vec{n}}^{2}}\right]\right)-i\left(\rho-\epsilon^{2} \sum_{\vec{n}>0} \pi \delta\left(m_{h}^{2}-m_{\vec{n}}^{2}\right)\right) .
\end{aligned}
$$

From this result we see that the Higgs mass renormalization is given by

$$
m_{h^{\prime}}^{2} \equiv m_{h}^{2}\left(1+\frac{\epsilon^{2}}{m_{h}^{2}} \Re\left[\sum_{\vec{n}>0} \frac{1}{m_{h}^{2}-i \rho-m_{\vec{n}}^{2}}\right]\right)
$$

in agreement with Eq. (17) of Ref. [7].

[1] L. Randall and R. Sundrum, Phys. Rev. Lett. 83, 3370 (1999), [hep-ph/9905221].

[2] D. Dominici, B. Grzadkowski, J. F. Gunion and M. Toharia, Nucl. Phys. B671, 243 (2003), [hep-ph/0206192].

[3] J. L. Hewett and T. G. Rizzo, JHEP 08, 028 (2003), [hep-ph/0202155].

[4] M. Battaglia, S. De Curtis, A. De Roeck, D. Dominici and J. F. Gunion, Phys. Lett. B568, 92 (2003), [hep-ph/0304245].

[5] I. Antoniadis, N. Arkani-Hamed, S. Dimopoulos and G. R. Dvali, Phys. Lett. B436, 257 (1998), [hep-ph/9804398].

[6] N. Arkani-Hamed, S. Dimopoulos and G. R. Dvali, Phys. Lett. B429, 263 (1998), [hep-ph/9803315].

[7] G. F. Giudice, R. Rattazzi and J. D. Wells, Nucl. Phys. B595, 250 (2001), [hep-ph/0002178].

[8] J. D. Wells, hep-ph/0205328.

[9] A. Datta, K. Huitu, J. Laamanen and B. Mukhopadhyaya, Phys. Rev. D70, 075003 (2004), [hep-ph/0404056].

[10] M. Battaglia, D. Dominici, J. F. Gunion and J. D. Wells, hep-ph/0402062.

[11] J. F. Gunion, L. Poggioli, R. J. Van Kooten, C. Kao and P. Rowson, Snowmass Workshop, 1996, hep-ph/9703330. 